\title{
Medical cloud platform for efficient flow control technology Fan Xincan ${ }^{1, \text { a }}$ \\ ${ }^{1}$ school of computer engineering, Shenzhen PolyTechnic, Shenzhen 518055,China \\ ahorse_fxc@163.com
}

Keywords: Medical cloud platform; flow control; Token bucket; Leaky bucket

\begin{abstract}
In this paper, cloud computing application in the construction of medical information technology, medical cloud platform for large-scale data needs to be efficient flow control, this paper first of several flow control algorithm, algorithm analysis, medical cloud platform the HTB flow control algorithm is applied, experimentalprove its cloud platform, data-processing capacity in the health care has improved significantly, and can maintain good stability.
\end{abstract}

\section{Introduction}

\section{Medical cloud platform}

In 2009 the country will" build up practical sharing medical information system" as the new health care reform plan four system construction supporting system, at the policy level to further promote the medical and health institutions of information construction. To crack the grass-roots medical institutions of the informationization construction difficult problem, the key point is that the grassroots medical institutions information construction mode, especially based on cloud computing software application architecture construction ${ }^{[1]}$. Cloud based low cost sharing platform adopts the centralized system, compared with the traditional construction mode has obvious advantages, it can be managed a variety of different applications, according to the needs of dynamic deployment, configuration and recovery of computer resources, real-time monitoring resource usage, to the vast number of front end users with sharing of computing resources and services. Flow control module is medical cloud platform is an important part of the huge amount of data, the platform, multiple and complex, the need for all kinds of different grade users were differentiated service, which requires high performance of flow control.

\section{Flow control technology}

Flow control technology, through the control of network traffic to certain data flow assurance or optimize the basic performance, reduce delay, the actual increase in available bandwidth and other functions, can be based on the specific data packet delay sending the realization. For a link, if the bearer data stream size exceeds a certain limit, the delay will increase significantly, it needs to meet a predetermined specific rules in data packet set to take certain delay strategy, which belongs to the traffic shaping ( Traffic Shaping ) category, the idea is for the particular data stream measurement and in case of need to delay, so that each data packet satisfies the implementation strategy. Every one needs to measure the data flow comprising a separate cache, the data stream message or cells are temporarily stored in the buffer, when calculating the packet or cell that meet the control strategy, will be issued. ${ }^{[2]}$

\section{Flow control algorithm}

\section{Flow control algorithm}

Token bucket algorithm is a network traffic shaping ( Traffic Shaping) and rate limit ( Rate Limiting ) in the most common usage of an algorithm. Typically, a token bucket algorithm is used to control the transmitting the data into the network number, and allow the burst data transmission. Token bucket this control mechanism based on token bucket are tokens to indicate when can send flow. Token bucket in each of a plurality of tokens are represented by one byte. If the token bucket in the presence of a token, is allowed to send flow; and if the token bucket does not exist in the 
token, are not allowed to send traffic.

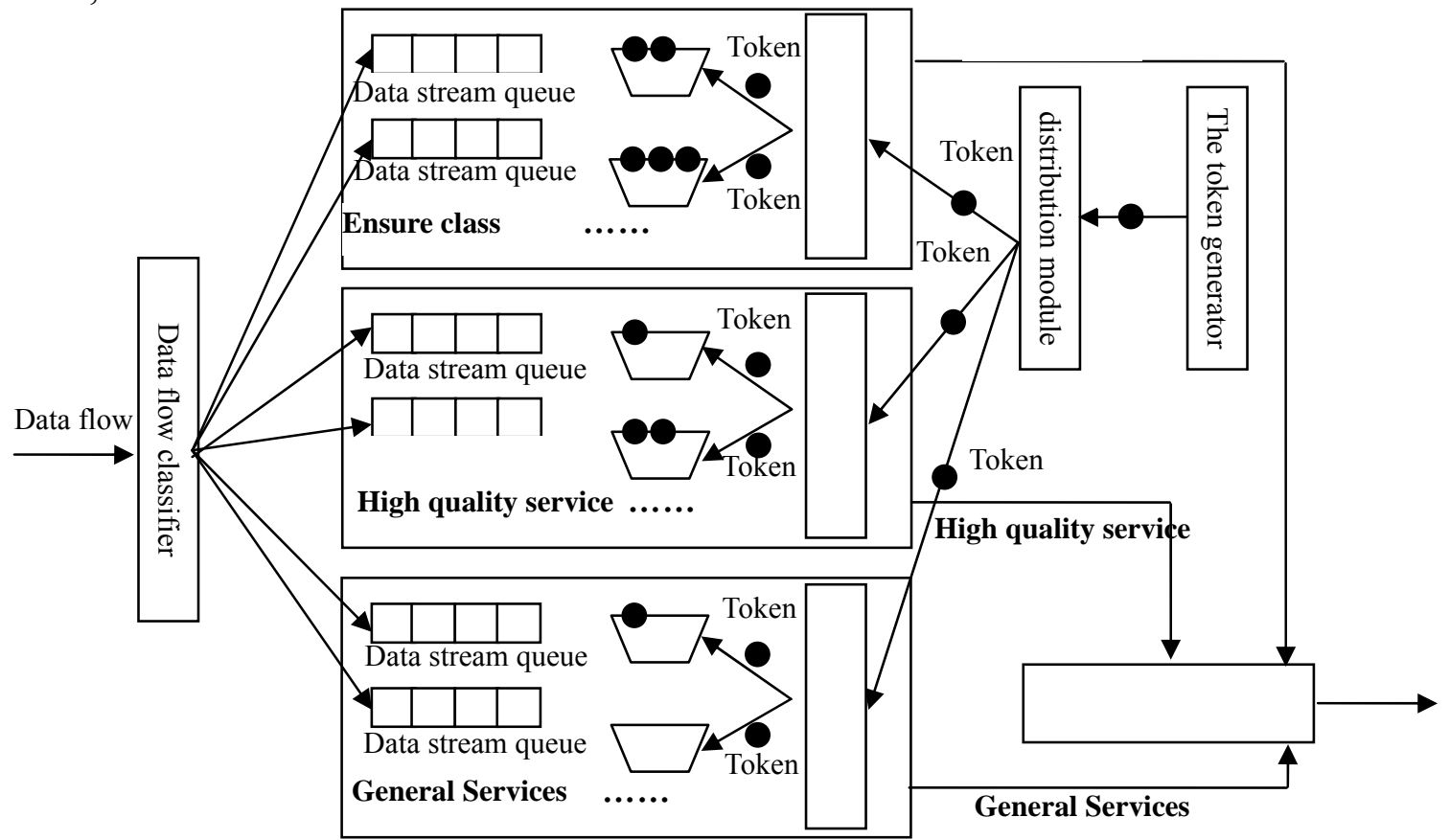

Fig.1Schematic diagram of the token bucket algorithm

As shown in Figure 1, flow classifier is responsible for the input flow by application of category classification after the cache to the correct categories and data flow waiting in the queue sends the token, output scheduling module according to the data stream queue acquired token number and queue filling state to judge whether the output of the queue in the data stream, the token by token and the reasonable distribution of distribution mechanism regulation of each service class and data flow the bandwidth resources.

Token bucket algorithm process available the following steps show: ${ }^{[3]}$

1) the assumption that each token represents the length of a byte, if the user configuration of the average transmission rate is $\mathrm{r}$, then every $1 / \mathrm{r}$ seconds a token is added to the bucket;

2) barrel maximum can store B token if the token bucket to seasonal card is full, so this token will be discarded;

3) when an $\mathrm{N}$ bytes of the packet arrival time from the token bucket, delete $\mathrm{n}$ token, and the packet is sent out;

4) if the token bucket in less than $n$ token, so not to remove the token, and that the data packet is sent to meet the conditions.

\section{Leaky bucket algorithm}

Leaky bucket algorithm is in the network world traffic shaping or rate limiting is often used when an algorithm, and its main purpose is to control data into the network speed, smoothness of the burst traffic in a network. Leaky bucket algorithm provides a mechanism, through it, bursty traffic can be plastic so as to provide a steady flow of network. Leaky bucket can be regarded as a constant business hours single server queue, if the leaky bucket ( packet buffer overflow ), then the packet will be discarded.

Algorithm for the basic contents are as follows ${ }^{[4]}$ :

1) forced a constant output rate regardless of the input data stream to the sudden. When the input is idle, the algorithm does not perform any action;

2) at each time slice to the network sends a packet, resulting in a fixed data rate, smooth traffic burst;

3) when the data packet with the same size ( such as ATM cells), each time a packet is sent work mechanism without any problems. But for variable length packets, each time slice can send the same as the number of data packets, but preferably sent a fixed number of bytes. For example: if each time slice transmission of 1024 bytes, so a time slice is allowed to transmit a 1024 byte packet, two 512 bytes of the packet, or four 256 bytes of the packet. 


\section{Medical cloud platform HTB algorithm application}

\section{HTB}

HTB ( Hierarchical Tokens Bucket ) is a widely used flow control mechanism, for different levels of the user's bandwidth requirements to undertake optimizing configuration, the bandwidth allocation, management, shaping, speed, user classification, bandwidth utilization factor, to meet the medical cloud platform features. HTB through the token bucket algorithm traffic shaping, based on different queue scheduling for each data flow speed, so as to achieve the overall classification link bandwidth, various kinds of control data flow rate to export. Compared with the traditional traffic control methods, HTB can not only according to certain ratio division and limitations of each particular data stream bandwidth, but also introduced" bandwidth borrowing" this new idea, namely allow data flow in bandwidth is idle when borrowing is allocated to other data flow with wide bandwidth, thus realizing the full and effective use of ${ }^{[5]}$.

\section{The principle of HTB algorithm}

\section{Concept description}

- Class: each class has to determine the rate of AR, CR, P, priority ceiling rate of total Q and level of level several parameters, the actual rate was characterized by $\mathrm{R}$.

- Leaf nodes: a leaf node bandwidth allocation unit is real, no offspring, only the leaf nodes in order to load a data packet queue, a leaf node is a leaf node in a logical hierarchy combinations.

Hierarchy: each leaf node has its own hierarchy of level, leaf nodes of level 0 , and the root node level for the total number of plies of minus 1 , intermediate nodes from the leaf nodes start layer-by-layer additive.

State: each leaf node has its own current state, i.e. class state, this state is from the category of $\mathrm{R}, \mathrm{AR}$ and $\mathrm{CR}$ values are calculated, there are red, yellow, green three:

\section{Red: $\mathrm{R}>\mathrm{CR}$}

Yellow: $\mathrm{AR}<\mathrm{R} \leq \mathrm{CR}$

Green: $\mathrm{R} \leq \mathrm{AR}$

$\mathrm{D}(\mathrm{C})$ : if a class $\mathrm{C}$ state of yellow, then $\mathrm{D}$ ( C ) refers to all the leaf nodes to transmit dataThe $\mathrm{C}$ offspring, as well as in these offspring and $\mathrm{C}$ between nodes. In short, just want to borrow from the class $\mathrm{C}$ beltWide node.

\section{Link sharing}

HTB link shared object defines each class of the actual rate of $\mathrm{R}$ calculation method. For a class of $\mathrm{C}$, the actual rate ${ }^{[6]}: R_{c}=\min =\left(C R_{c}, A R_{c}+B_{c}\right)$ node ):

Where $B_{c}$ is from the ancestral borrow bandwidth, can be expressed as ( $\mathrm{P}$ C parent

$$
B_{C}=\left\{\begin{array}{l}
\frac{Q_{C} \times R_{p}}{\sum Q_{i} \in D(p) Q_{i}}, \min \left(p_{i} \in D_{p}\right) \geq p_{c} \\
0, \text { Others }
\end{array}\right.
$$

As can be seen from the formula, when the bandwidth sharing class if there is no parent node, $B_{c}$ is 0 , if offspring to send queue has a higher priority class, should first serve them.

\section{Implementation scheduling scheme}

As shown in Figure 2, HTB is described in the scheduling process, HTB classes in the flow dividing is free to organize tree structure, but in the real implementation, class of transmit and use a relationship is based on the red-black tree form of organization and management. The existence of a global tree structure of self slots, every level, every priority uniquely corresponds to a self of slot, every internal leaf class has a set of inner feed slots. If all classes from the correct structure of management, only need to select a data packet self slot levels lowest, highest priority. 


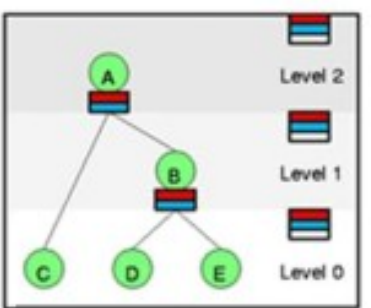

1

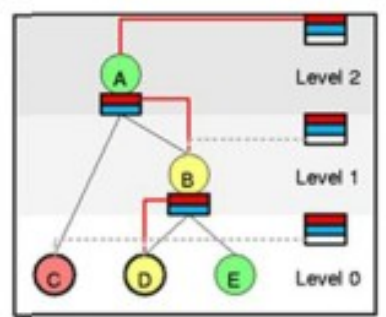

4

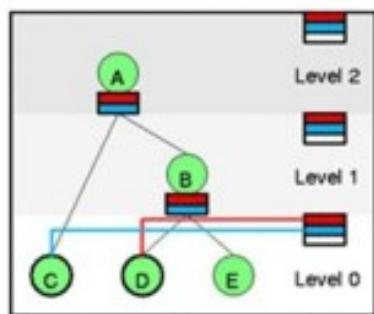

2

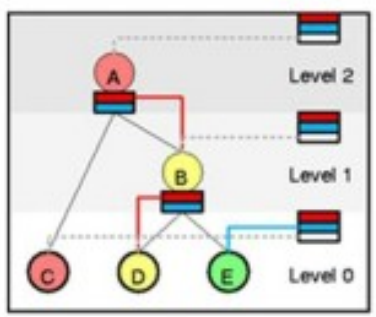

5

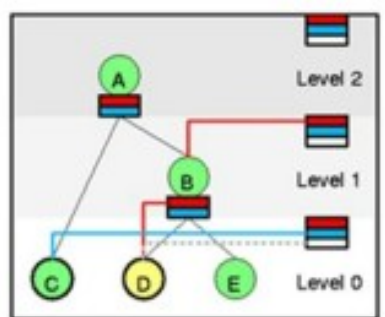

3

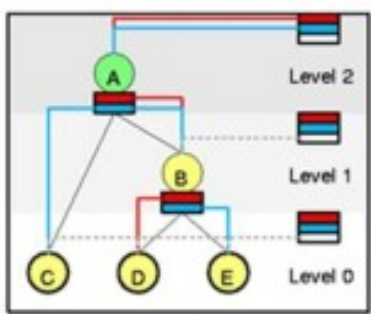

6

Fig.2 Schematic diagram of HTB scheduling

\section{Experiment and Summary}

In the medical cloud platform design of scene features a needle to export HTB bandwidth allocation of tree, tree leaf node represents a real need to be assigned to the actual bandwidth for different types of services. Test design of two types of service:0.5Mbps/1Mbps and $2 \mathrm{Mbps} / 12 \mathrm{Mbps}$. Experiments using the user scale pressure test, used in the experiments and mutex queue as a data packet queue testing, more than 8001600 user node, service node, verification of parallel optimization HTB in medical cloud platform actual scenes of mass user and data flow can work.

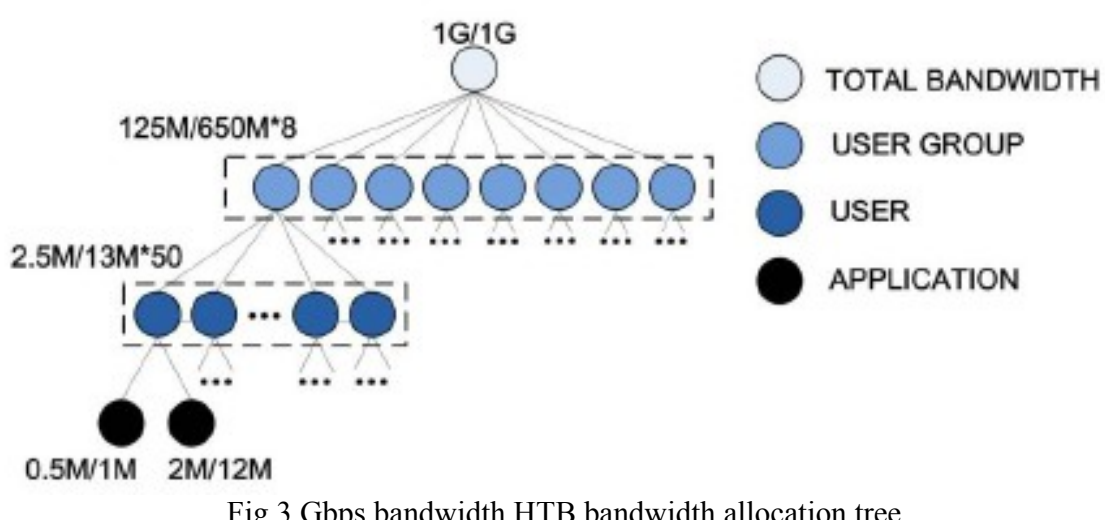

Fig.3 Gbps bandwidth HTB bandwidth allocation tree

Proved by experiments, the HTB algorithm in medical cloud platform flow control module application significantly reduced the extra time consumption, accelerate the access speed, and the data flow pressure is greater, but also has good stability. Using the cloud platform a powerful storage resources, computing resources and network resources, based on cloud computing and efficient medical information service, for efficient medical information service application, for each hospital information construction can produce huge economy and social benefit.

\section{Reference}

[1] Chia-Chi Teng, Jonathan Mitchell, Christopher Walker.A medical image archive solution in the cloud.Software Engineering and Service Sciences (ICSESS), 2010 IEEE International Conference on [2] Carlos Oberdan Rolim, Fernando Luiz Koch, Carlos Becker Westphall.A Cloud Computing Solution for Patient's Data Collection in Health Care Institutions.eHealth, Telemedicine, and Social Medicine, 2010. ETELEMED '10. Second International Conference on

[3] Anuj Kumar Yadav,Ravi Tomar,Deep Kumar,Himanshu Gupta.Security and Privacy Concerns in Cloud Computing.Computer Science and Software Engineering.Volume 2, Issue 5, May 2012 
[4] Valenzuela, J.L. A hierarchical token bucket algorithm to enhance QoS in IEEE 802.11: proposal, implementation and evaluation.Vehicular Technology Conference, 2004. VTC2004-Fall. 2004 IEEE 60 th

[5] Soranun Jiwasurat.George Kesidis.David J. Miller.Hierarchical shaped deficit round-robin scheduling.Global Telecommunications Conference, 2005. GLOBECOM '05. IEEE

[6] Yi FaSheng,A method based on resource management end-system traffic shaping algorithm [J]. computer engineering and application of.2007(18):117-119 